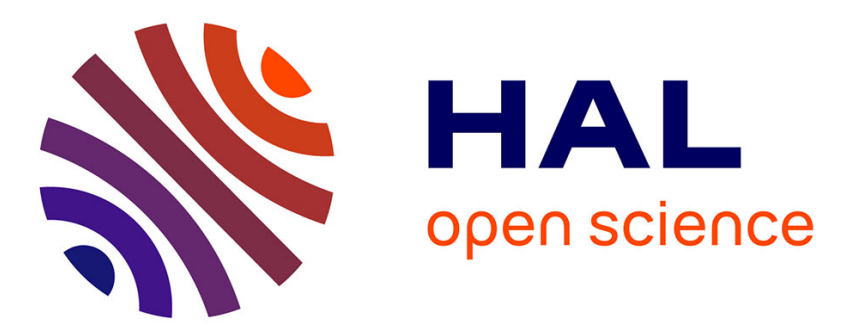

\title{
Numerical study of the fracture diffusion-dispersion coefficient for passive transport in fractured porous media
}

Florent Chave

\section{- To cite this version:}

Florent Chave. Numerical study of the fracture diffusion-dispersion coefficient for passive transport in fractured porous media. Numerical Mathematics and Advanced Applications ENUMATH 2019, Sep 2019, Egmond aan Zee, Netherlands. hal-02412691v2

\section{HAL Id: hal-02412691 \\ https://hal.science/hal-02412691v2}

Submitted on 20 Apr 2020

HAL is a multi-disciplinary open access archive for the deposit and dissemination of scientific research documents, whether they are published or not. The documents may come from teaching and research institutions in France or abroad, or from public or private research centers.
L'archive ouverte pluridisciplinaire HAL, est destinée au dépôt et à la diffusion de documents scientifiques de niveau recherche, publiés ou non, émanant des établissements d'enseignement et de recherche français ou étrangers, des laboratoires publics ou privés. 


\title{
Numerical study of the fracture diffusion-dispersion coefficient for passive transport in fractured porous media
}

Florent Chave

\begin{abstract}
We propose a new definition of the normal fracture diffusion-dispersion coefficient for a reduced model of passive transport in fractured porous media.
\end{abstract}

Key words: Fractured porous media, passive transport, Hybrid High-Order, nonconforming methods, polyhedral meshes, arbitrary order

MSC (2010): 65N08, 65N12, 65N30

\section{Introduction}

In this paper, we focus on the reduced model introduced in $[1,3]$ describing the Passive Transport of a solute in a Fractured Porous Media, which will be now refered to as (PTFPM). By reduced model, we assume that the fracture is treated as a surface of codimension one. The reduced model (PTFPM) consists of two advectiondiffusion-reaction equations, one in the porous media and one in the fracture, with advective velocity fields taken as the solution of a decoupled problem, and where the coupling is done by subtle transmission conditions describing the exchanges between the different regions. A notable feature of the reduced model (PTFPM) is that the transmission conditions between the porous media and the fracture mimic at the discrete level the property that the advection terms do not contribute to the energy balance of the system, allowing us to handle both conducting and blocking fractures by letting the concentration of the solute jumps across the fracture; see also [7] in the context of advection of a passive scalar in a fractured porous media. However, the description of the fracture diffusion-dispersion in both the normal and tangential directions considered in the reduced model (PTFPM) is meaningless

Florent Chave

Inria, Univ. Lille, CNRS, UMR 8524 - Laboratoire Paul Painlevé, F-59000 Lille, France

e-mail: florent.chave@inria.fr 
from the physical viewpoint. Indeed, in (PTFPM) those coefficients are assumed to be independent from the surrounding unknowns: this is irrelevent since they play an important role in the description of (i) the exchanges between the porous media and the fracture, and (ii) the behavior of the solute at the neighborhood of the fracture. The aim of this paper is to propose a more physical definition of the fracture diffusion-dispersion coefficient, and to present some test cases based on the previous works $[2,3]$. The rest of this paper is organized as follows: in Section 2 we present the main equations and in Section 3 we perform numerical experiments.

\section{The differential model}

In this section, we present the reduced model for the passive transport in a fractured porous media. We first introduce notation, then define the velocity fields and diffusion-dispersion tensors, and finally introduce the main equations.

\subsection{Notation}

We consider a porous medium saturated by an incompressible fluid that occupies a space region $\Omega \subset \mathbb{R}^{2}$ traversed by a fracture $\Gamma$. We assume that $\Omega$ is an open, bounded, connected, polygonal set with Lipschitz boundary $\partial \Omega$, and denote by $\mathbf{n}_{\partial \Omega}$ the unit normal vector on $\partial \Omega$ pointing out of $\Omega$. The fracture $\Gamma$ is represented by an open line segment of nonzero length which cuts $\Omega$ into two disjoint connected polygonal subdomains $\Omega_{\mathrm{B}, 1}$ and $\Omega_{\mathrm{B}, 2}$ with Lipschitz boundary. The sets $\Omega_{\mathrm{B}}:=\Omega \backslash$ $\bar{\Gamma}=\Omega_{\mathrm{B}, 1} \cup \Omega_{\mathrm{B}, 2}$ and $\partial \Omega_{\mathrm{B}}:=\cup_{i+1}^{2}\left(\partial \Omega_{\mathrm{B}, i} \backslash \bar{\Gamma}\right)$ correspond to the bulk region and the external boundary of the bulk region, respectively. The boundary of the fracture $\Gamma$ is denoted by $\partial \Gamma$, and the corresponding outward unit tangential vector is $\tau_{\partial \Gamma}$. Finally, $\mathbf{n}_{\Gamma}$ denotes the unit normal vector to $\Gamma$ pointing out of $\Omega_{\mathrm{B}, 1}$ This notation is illustrated in Figure 1.

Fig. 1 Illustration of the notation introduced in Section 2.1.

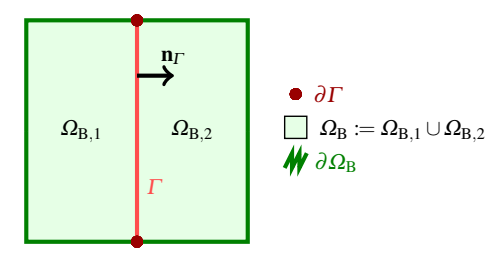

For any scalar- or vector-valued function $\varphi$ sufficiently regular to admit a (possibly two-valued) trace on $\Gamma$, we define the jump and average operators such that

$$
\llbracket \varphi]_{\Gamma}:=\left(\varphi_{\mid \Omega_{\mathrm{B}, 1}}-\varphi_{\mid \Omega_{\mathrm{B}, 2}}\right)_{\mid \Gamma}, \quad\{\{\varphi\}\}_{\Gamma}:=\frac{1}{2}\left(\varphi_{\mid \Omega_{\mathrm{B}, 1}}+\varphi_{\mid \Omega_{\mathrm{B}, 2}}\right)_{\mid \Gamma} .
$$




\subsection{Advective velocity fields}

We assume that the advective Darcy velocities follow from the decoupled reduced model [8], which describes the flow in a fractured porous medium. This model reads as follows: Find the bulk Darcy velocity $\mathbf{u}: \Omega_{\mathrm{B}} \rightarrow \mathbb{R}^{2}$, the bulk pressure $p: \Omega_{\mathrm{B}} \rightarrow \mathbb{R}$ and the fracture pressure $p_{\Gamma}: \Gamma \rightarrow \mathbb{R}$ such that

$$
\begin{aligned}
\mathbf{u}+\mathbf{K} \nabla_{p} & =0 & & \text { in } \Omega_{\mathrm{B}}, \\
\nabla \cdot \mathbf{u} & =f & & \text { in } \Omega_{\mathrm{B}}, \\
\mathbf{u} \cdot \mathbf{n}_{\partial \Omega} & =0 & & \text { on } \partial \Omega_{\mathrm{B}}, \\
\nabla_{\tau} \cdot\left(-K_{\Gamma} \nabla_{\tau} p_{\Gamma}\right) & =\ell_{\Gamma} f_{\Gamma}+\left[[\mathbf{u}]_{\Gamma} \cdot \mathbf{n}_{\Gamma}\right. & & \text { in } \Gamma, \\
-K_{\Gamma} \nabla_{\tau} p_{\Gamma} \cdot \tau_{\partial \Gamma} & =0 & & \text { on } \partial \Gamma, \\
\int_{\Gamma} p_{\Gamma} & =0, & &
\end{aligned}
$$

where $f \in L^{2}\left(\Omega_{\mathrm{B}}\right)$ and $f_{\Gamma} \in L^{2}(\Gamma)$ verify $\int_{\Omega_{\mathrm{B}}} f+\int_{\Gamma} \ell_{\Gamma} f_{\Gamma}=0$ and denote source or sink terms, $\mathbf{K}: \Omega_{\mathrm{B}} \rightarrow \mathbb{R}^{2 \times 2}$ is the bulk permeability tensor, and we have set $K_{\Gamma}:=$ $\kappa_{\Gamma}^{\tau} \ell_{\Gamma}$, with $\kappa_{\Gamma}^{\tau}: \Gamma \rightarrow \mathbb{R}$ denoting the tangential permeability inside the fracture and $\ell_{\Gamma}: \Gamma \rightarrow \mathbb{R}$ the fracture thickness. In (1d) and (1e), $\nabla_{\tau}$ and $\nabla_{\tau} \cdot$ denote the tangential gradient and divergence operators along $\Gamma$, respectively. The following transmission conditions across the fracture close the problem:

$$
\{\{\mathbf{u}\}\}_{\Gamma} \cdot \mathbf{n}_{\Gamma}=\frac{\kappa_{\Gamma}^{n}}{\ell_{\Gamma}} \llbracket[p]_{\Gamma} \text { on } \Gamma, \quad\left[[\mathbf{u}]_{\Gamma} \cdot \mathbf{n}_{\Gamma}=\frac{\kappa_{\Gamma}^{n}}{\ell_{\Gamma}} \xi^{-1}\left(\{\{p\}\}_{\Gamma}-p_{\Gamma}\right) \text { on } \Gamma,\right.
$$

where $\xi \in\left(0, \frac{1}{2}\right]$ is a user-dependent model parameter and $\kappa_{\Gamma}^{n}: \Gamma \rightarrow \mathbb{R}$ represents the normal permeability inside the fracture. From now, we refer to the advective velocity fields as the bulk Darcy velocity $\mathbf{u}$ and the tangential fracture Darcy velocity $\mathbf{u}_{\Gamma}:=-K_{\Gamma} \nabla_{\tau} p_{\Gamma}$.

\subsection{Diffusion-dispersion tensors}

Following [9], we assume that the bulk diffusion-dispersion tensor $\mathbf{D}: \Omega_{\mathrm{B}} \rightarrow \mathbb{R}^{2 \times 2}$ and the fracture diffusion-dispersion coefficient $D_{\Gamma}: \Gamma \rightarrow \mathbb{R}$ are such that

$$
\begin{aligned}
\mathbf{D} & :=\phi\left(d_{\mathrm{m}} \mathbf{I}_{2}+|\mathbf{u}|\left(d_{1} \mathbf{E}(\mathbf{u})+d_{\mathrm{t}}\left(\mathbf{I}_{2}-\mathbf{E}(\mathbf{u})\right)\right)\right), \\
D_{\Gamma} & :=\phi_{\Gamma}\left(\ell_{\Gamma} d_{\mathrm{m}}^{\Gamma}+\left|\mathbf{u}_{\Gamma}\right| d_{1}^{\Gamma}\right),
\end{aligned}
$$

where $\mathbf{u}$ and $\mathbf{u}_{\Gamma}$ are defined in Section 2.2, $|\cdot|$ is the euclidian norm, and the scalar functions $\phi, d_{\mathrm{m}}, d_{1}, d_{\mathrm{t}}: \Omega \rightarrow \mathbb{R}$ and $\phi_{\Gamma}, d_{\mathrm{m}}^{\Gamma}, d_{1}^{\Gamma}: \Gamma \rightarrow \mathbb{R}$ are, respectively, the bulk porosity, molecular diffusion, longitudinal and transverse dispersion coefficients, and the fracture porosity, molecular diffusion and longitudinal dispersion coeffi- 
cients. In (3a), $\mathbf{I}_{2} \in \mathbb{R}^{2 \times 2}$ is the identity matrix and $\mathbf{E}(\mathbf{u}):=|\mathbf{u}|^{-2}(\mathbf{u} \otimes \mathbf{u}) \in \mathbb{R}^{2 \times 2}$ denotes the orthogonal projection matrix in the direction of $\mathbf{u}$. In the reduced model (PTFPM), the fracture diffusion-dispersion coefficient $D_{\Gamma}$ depends on a fracture transverse dispersion coefficient. Here, the fracture transverse dispersion coefficient is rather integrated into the transmission conditions; see Remark 1.

\subsection{The reduced model}

For a fixed $T>0$, we denote by $\Omega_{\mathrm{B}}^{T}:=(0, T) \times \Omega_{\mathrm{B}}$ and $\Gamma^{T}:=(0, T) \times \Gamma$ the temporal-spatial domains of interest, and by $\partial \Omega_{\mathrm{B}}^{T}:=(0, T) \times \partial \Omega_{\mathrm{B}}$ and $\partial \Gamma^{T}:=$ $(0, T) \times \Gamma$ their respective boundaries. The reduced model for the passive transport of a solute in a fractured porous medium hinges into seeking the concentration of the solute in the bulk $c: \Omega^{T} \rightarrow \mathbb{R}$ and in the fracture $c_{\Gamma}: \Gamma^{T} \rightarrow \mathbb{R}$ such that

$$
\begin{array}{rlrl}
\phi \partial_{t} c+\nabla \cdot(\mathbf{u} c-\mathbf{D} \nabla c)+f^{-} c & =f^{+} \widehat{c} & & \text { in } \Omega_{\mathrm{B}}^{T}, \\
-\mathbf{D} \nabla c \cdot \mathbf{n}_{\partial \Omega} & =0 & & \text { on } \partial \Omega_{\mathrm{B}}^{T}, \\
\ell_{\Gamma} \phi_{\Gamma} \partial_{t} c_{\Gamma}+\nabla_{\tau} \cdot\left(\mathbf{u}_{\Gamma} c_{\Gamma}-D_{\Gamma} \nabla_{\tau} c_{\Gamma}\right)+\ell_{\Gamma} f_{\Gamma}^{-} c_{\Gamma} & =\ell_{\Gamma} f_{\Gamma}^{+} \widehat{c_{\Gamma}} & & \text { in } \Gamma^{T}, \\
-D_{\Gamma} \nabla_{\tau} c_{\Gamma} \cdot \tau_{\partial \Gamma} & =0 & & +\llbracket \mathbf{u} c-\mathbf{D} \nabla c]_{\Gamma} \cdot \mathbf{n}_{\Gamma} \\
& & \text { on } \partial \Gamma^{T},
\end{array}
$$

where $\mathbf{u}$ and $\mathbf{u}_{\Gamma}$ are defined in Section 2.2, $\mathbf{D}$ and $D_{\Gamma}$ are defined in Section 2.3, the terms $f^{ \pm}:=1 / 2(|f| \pm f)$ and $f_{\Gamma}^{ \pm}:=1 / 2\left(\left|f_{\Gamma}\right| \pm f_{\Gamma}\right)$ denote the positive or negative part of $f$ and $f_{\Gamma}$, respectively, and the scalar functions $\widehat{c}: \Omega_{\mathrm{B}}^{T} \rightarrow \mathbb{R}$ and $\widehat{c_{\Gamma}}: \Gamma^{T} \rightarrow \mathbb{R}$ stand for the concentration of solute as it is injected in the bulk and in the fracture, respectively. The following transmission conditions, along with initial conditions $c(t=0)=c_{0}$ in $\Omega_{\mathrm{B}}$ and $c_{\Gamma}(t=0)=c_{\Gamma, 0}$ in $\Gamma$, close the problem:

$$
\begin{aligned}
\{\{\mathbf{u} c-\mathbf{D} \nabla c\}\}_{\Gamma} \cdot \mathbf{n}_{\Gamma} & \left.=\frac{\mathscr{D}_{\Gamma}^{n}}{\ell_{\Gamma}} \llbracket c\right] \rrbracket_{\Gamma}+\{\{c\}\}_{\Gamma}\{\{\mathbf{u}\}\}_{\Gamma} \cdot \mathbf{n}_{\Gamma}+\frac{1}{8} \llbracket c c \rrbracket_{\Gamma} \llbracket \mathbf{u} \rrbracket_{\Gamma} \cdot \mathbf{n}_{\Gamma} \text { on } \Gamma, \\
{\left[\mathbf{u} c-\mathbf{D} \nabla c \rrbracket_{\Gamma} \cdot \mathbf{n}_{\Gamma}\right.} & =\frac{\mathscr{D}_{\Gamma}^{n}}{\ell_{\Gamma}} \xi^{-1}\left(\{\{c\}\}_{\Gamma}-c_{\Gamma}\right)+\frac{1}{2}\left(\{\{c\}\}_{\Gamma}+c_{\Gamma}\right) \llbracket \mathbf{u} \rrbracket_{\Gamma} \cdot \mathbf{n}_{\Gamma} \text { on } \Gamma,
\end{aligned}
$$

where $\xi$ is the user-dependent model parameter introduced in Section 2.2. The term $\mathscr{D}_{\Gamma}^{n}: \Gamma \rightarrow \mathbb{R}$ represents the normal diffusion-dispersion coefficient of the fracture. In the reduced model (PTFPM), $\mathscr{D}_{\Gamma}^{n}$ does not depend on the surrounding unknowns. For a more accurate description of the exchange between the bulk and the fracture, we propose the following definition:

$$
\mathscr{D}_{\Gamma}^{n}:=\phi_{\Gamma}\left(d_{\mathrm{m}}^{\Gamma}+d_{\mathrm{t}}^{\Gamma}\left|\{\{\mathbf{u}\}\}_{\Gamma} \cdot \mathbf{n}_{\Gamma}\right|\right),
$$

that depends on (i) the porosity of the fracture $\phi_{\Gamma}$, (ii) the fracture molecular diffusion coefficient $d_{\mathrm{m}}^{\Gamma}$, and (iii) on the fracture transverse dispersion $d_{\mathrm{t}}^{\Gamma}: \Gamma \rightarrow \mathbb{R}$ 
weighted by the normal component of the average of the bulk Darcy velocity $\mathbf{u}$. From now, we refer to the reduced model (4)-(5)-(6) as (PTFPM $\star)$.

Remark 1. The fracture transverse dispersion $d_{\mathrm{t}}^{\Gamma}$ describes the property of the solute to diffuse in the orthogonal directions of the fracture advective velocity field $\mathbf{u}_{\Gamma}$. In the framework of reduced models, it is assumed that the normal componant of the fracture Darcy velocity is a linear combinaison of the normal component of the surrounding bulk Darcy velocity; see [8]. Therefore, it seems natural to integrate the fracture transverse dispersion coefficient into the transmission conditions (5).

\section{Numerical experiments}

In this section we numerically compare the two reduced models (PTFPM) and (PTFPM $\star$ ). For the sake of brevety, we refer to the previous works [2, 3] for the space discretization aspects and to [3, Section 5] for an in-depth description of the test case configurations considered in this section. To discretize in time, we use a backward Euler scheme and consider a uniform partition $\left(t^{n}\right)_{0 \leq n \leq N}$ of the time interval $(0, T)$ with $t^{0}=0, t^{N}=T$ and $t^{n}-t^{n-1}=\Delta t$ for all $1 \leq n \leq N$.

\subsection{Injection and production wells}

In petroleum engineering, the source terms $f$ and $f_{\Gamma}$ are used to model injection and production wells in the bulk and in the fracture, respectively; see [10]. Through this section, the injection well sits in $\mathbf{x}_{\mathrm{i}} \in \Omega_{\mathrm{B}}$, the production one in $\mathbf{x}_{\mathrm{p}} \in \Omega_{\mathrm{B}}$, and both are modeled by the source term $f$ defined such that

$$
f(\mathbf{x})=\frac{1}{2}\left(\tanh \left(200\left(0.025-\left|\mathbf{x}-\mathbf{x}_{\mathbf{i}}\right|\right)\right)-\tanh \left(200\left(0.025-\left|\mathbf{x}-\mathbf{x}_{\mathrm{p}}\right|\right)\right)\right) .
$$

For a fixed $T_{\mathrm{inj}}>0$, the concentration of solute as it is injected is defined as $\widehat{c}(t, \mathbf{x})=$ 1 if $t<T_{\text {inj }}$ and $\widehat{c}(t, \mathbf{x})=0$ otherwise. In the fracture, we set $f_{\Gamma} \equiv \widehat{c_{\Gamma}}(t, \mathbf{x}) \equiv 0$. We assume that the initial concentration of solute is zero in $\Omega_{\mathrm{B}}$ and $\Gamma$.

\subsection{Impermeable fractures}

We first consider a test case modelling the passive displacement of a solute in a fractured porous medium where fractures act as barriers. The domain configuration and user parameters are detailed in Figure 2a. With this configuration the solute is expected to go from the injection well toward the production well by avoiding the fractures; see [3, Section 5.2]. In Figures $2 \mathrm{~b}$ and 2c, we display the bulk concen- 
trations of both reduced models (PTFPM) and (PTFPM $\star$ ) obtained at different time $t$. In both cases, the solute follows the corridors designed by the fractures acting as barriers and goes from the injection to the production well for the two configurations. However, discontinuities of the bulk concentration $c$ across the fractures are more pronounced in the reduced model (PTFPM $\star$ ). This arises from the fact that the fracture transverse coefficient $d_{\mathrm{t}}^{\Gamma}$ depends on the surrounding bulk Darcy velocity, which, in this case, avoids fractures.

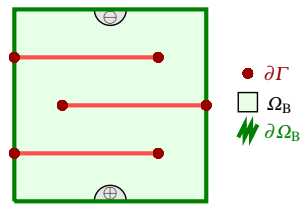

$$
\begin{aligned}
& \Omega=(0,1)^{2}, \xi=0.125, \mathbf{x}_{\mathrm{i}}=(1 / 2,0), \mathbf{x}_{\mathrm{p}}=(1 / 2,1) \\
& \Gamma=(0,3 / 4) \times\{1 / 4,3 / 4\} \cup(1 / 4,1) \times\{1 / 2\} \\
& \ell_{\Gamma}=10^{-2}, \mathbf{K}=10^{-3} \mathbf{I}_{2}, \boldsymbol{\kappa}_{\Gamma}^{\tau}=10^{-3}, \kappa_{\Gamma}^{n}=10^{-6} \\
& d_{\mathrm{m}}=d_{\mathrm{m}}^{\Gamma}=10^{-5}, d_{1}=d_{1}^{\Gamma}=1, d_{\mathrm{t}}=d_{\mathrm{t}}^{\Gamma}=10^{-2} \\
& \phi=\phi_{\Gamma}=10^{-1}, T=10^{2}, T_{\mathrm{inj}}=30, \Delta t=1
\end{aligned}
$$

(a) Domain configuration (left) and parameters (right)

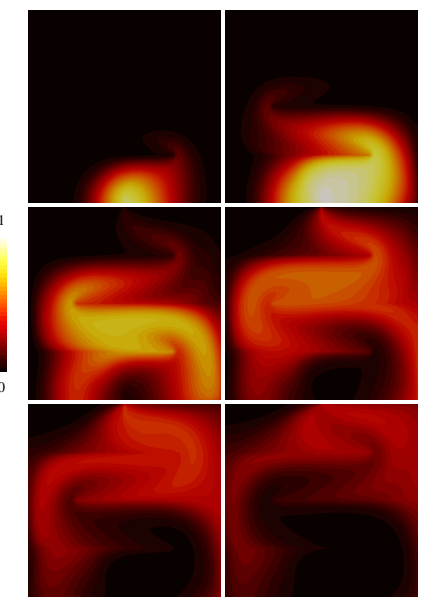

(b) Reduced model (PTFPM)

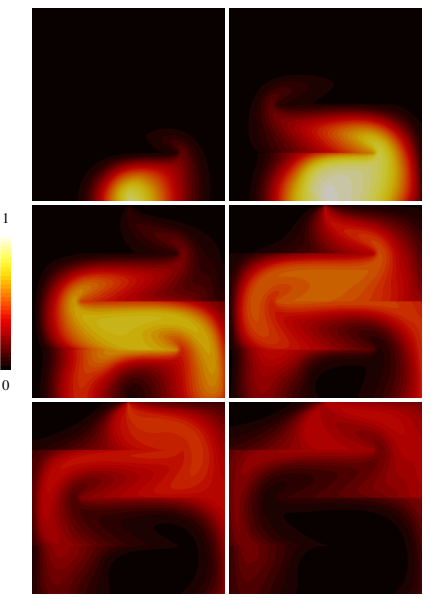

(c) Reduced model (PTFPM $\star$ )

Fig. 2 Domain configuration and parameters (top), and snapshots of the bulk concentrations $c$ (bottom) for the test case of Section 3.2 (impermeable fractures). Displayed times (from left to right, top to bottom): $t=5,20,40,60,80,100$.

\subsection{Permeable fractures}

We now consider fractures acting as conduits. Both the domain configuration and user parameters are displayed in Figure 3a. With this choice, it is expected that the solute is attracted by the fractures; see [3, Section 5.3]. In Figures $3 b$ and $3 c$, we display bulk concentrations $c$ of both reduced models (PTFPM) and (PTFPM $\star$ ), at different time $t$. In both cases, we can distinctly see that the solute channeled by the fractures flows towards the production well faster than the solute in the surrounding 
bulk medium. We remark that the discontinuities of the concentration $c$ are also in this case more pronounced at the neighbourhood of the fracture tips located near the injection well for the reduced model (PTFPM $\star$ ).

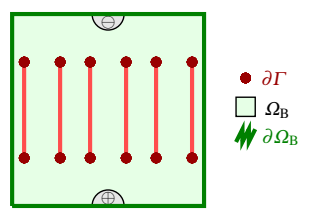

$$
\begin{aligned}
& \Omega=(0,1)^{2}, \xi=0.125, \mathbf{x}_{\mathrm{i}}=(1 / 2,0), \mathbf{x}_{\mathrm{p}}=(1 / 2,1) \\
& \Gamma=\{2 / 32,8 / 32,13 / 32,19 / 32,24 / 32,30 / 32\} \times(1 / 4,3 / 4) \\
& \ell_{\Gamma}=10^{-2}, \mathbf{K}=10^{-3} \mathbf{I}_{2}, \boldsymbol{K}_{\Gamma}^{\tau}=10^{-1}, \kappa_{\Gamma}^{n}=10^{-3} \\
& d_{\mathrm{m}}=d_{\mathrm{m}}^{\Gamma}=10^{-5}, d_{1}=d_{1}^{\Gamma}=1, d_{\mathrm{t}}=d_{\mathrm{t}}^{\Gamma}=10^{-2} \\
& \phi=\phi_{\Gamma}=10^{-1}, T=10^{2}, T_{\mathrm{inj}}=30, \Delta t=1
\end{aligned}
$$

(a) Domain configuration (left) and parameters (right)

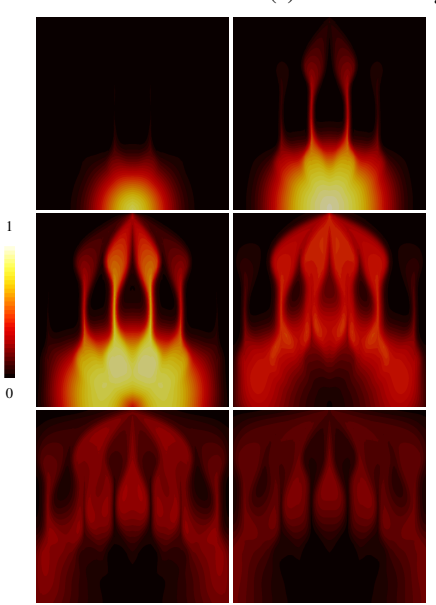

(b) Reduced model (PTFPM)

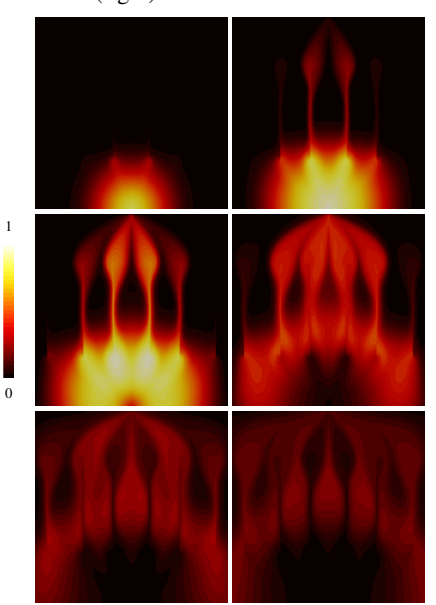

(c) Reduced model (PTFPM $\star)$

Fig. 3 Domain configuration and parameters (top), and snapshots of the bulk concentrations $c$ (bottom) for the test of Section 3.3 (permeable fractures). Displayed times (from left to right, top to bottom): $t=5,15,30,50,80,100$.

In practice, the molecular diffusion coefficients are set to zero. This delicate case is prone to instabilities since the diffusion-dispersion tensors can be degenerate in some parts of the domain where the Darcy velocities vanish. Moreover, the fracture normal diffusion-dispersion coefficient depends, in this case, only on the Darcy velocity u. In Figure 4, we display the concentrations obtained by the two reduced models (PTFPM) and (PTFPM $\star$ ) at different time $t$ upon setting $d_{\mathrm{m}}=d_{\mathrm{m}}^{\Gamma}=0$. Clearly, one can see instabilities at the neighborhood of the fractures for the reduced model (PTFPM); see Figure 4a. On the other hand, the reduced model (PTFPM $\star$ ) seems to handle without difficulty this particular case; see Figure $4 \mathrm{~b}$. We also note that the discontinuities are more pronounced in the reduced model (PTFPM $\star$ ), and that the concentrations of the two reduced models (PTFPM) and (PTFPM $\star$ ) behave differently at the vicinity of the fractures. 


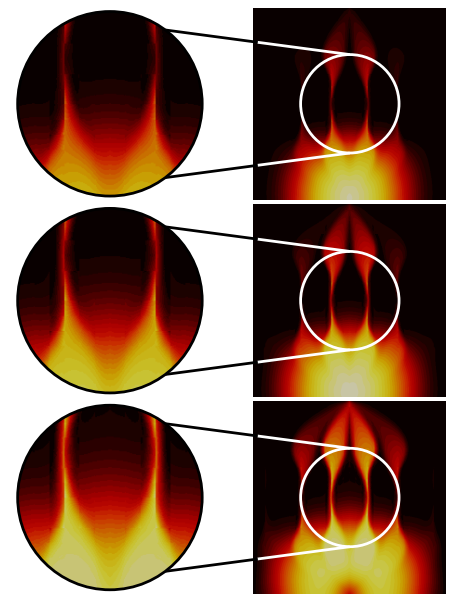

(a) Reduced model (PTFPM)

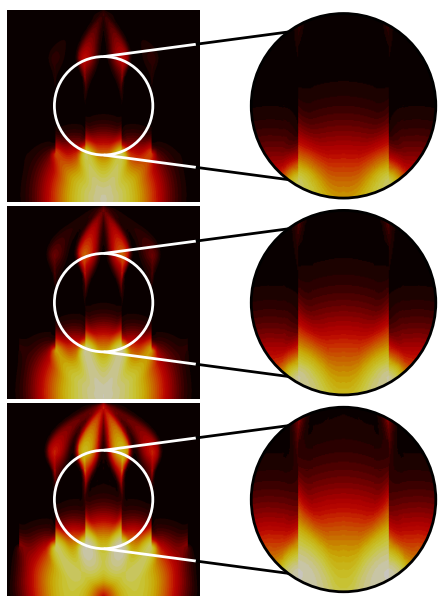

(b) Reduced model $(\mathrm{PTFPM} \star)$

Fig. 4 Snapshots of the bulk concentration $c$ and zoom on the vicinity of the fracture for the test case of Section 3.3 (permeable fracture, vanishing molecular diffusion). Displayed times: $t=$ $15,20,30$.

\section{References}

1. Chave, F.: Hybrid High-Order methods for interface problems. Ph.D. thesis, University of Montpellier, France and Polytechnic University of Milan, Italy (2018). URL https://tel.archives-ouvertes.fr/tel-01926061v2/document

2. Chave, F., Di Pietro, D.A., Formaggia, L.: A Hybrid High-Order method for Darcy flows in fractured porous media. SIAM J. Sci. Comput. 40(2), A1063-A1094 (2018). DOI $10.1137 / 17 \mathrm{M} 1119500$

3. Chave, F., Di Pietro, D.A., Formaggia, L.: A Hybrid High-Order method for passive transport in fractured porous media. Int. J. Geomath. 10(12) (2019). DOI 10.1007/s13137-019-0114-x

4. Di Pietro, D.A., Droniou, J.: The Hybrid High-Order method for polytopal meshes. No. 19 in Modeling, Simulation and Applications. Springer International Publishing (2020). DOI 10.1007/978-3-030-37203-3

5. Di Pietro, D.A., Ern, A.: A Hybrid High-Order locking-free method for linear elasticity on general meshes. Comput. Methods Appl. Mech. Engrg. 283, 1-21 (2015). DOI 10.1016/j.cma.2014.09.009

6. Di Pietro, D.A., Ern, A., Lemaire, S.: An arbitrary-order and compact-stencil discretization of diffusion on general meshes based on local reconstruction operators. Comput. Methods Appl. Math. 14(4), 461-472 (2014). DOI 10.1515/cmam-2014-0018

7. Fumagalli, A., Keilegavlen, E.: Dual virtual element methods for discrete fracture matrix models. Oil Gas Sci. Technol. - Rev. IFP Energies nouvelles 74(41) (2019). DOI 10.2516/ogst/2019008

8. Martin, V., Jaffré, J., Roberts, J.E.: Modeling fractures and barriers as interfaces for flow in porous media. SIAM J. Matrix Analysis and Applications 26(5), 1667-1691 (2005). DOI 10.1137/S1064827503429363

9. Peaceman, D.W.: Improved treatment of dispersion in numerical calculation of multidimensional miscible displacement. Soc. Petrol. Eng. J. 6, 213-216 (1966). DOI 10.2118/1362-PA

10. Todd, M.R., O’Dell, P.M., Hirasaki, G.J.: Methods for increased accuracy in numerical reservoir simulators. Soc. Petrol. Eng. J. 12, 515-530 (1972). DOI 10.2118/3516-PA 